\title{
Thermal analysis of protruding surfaces in the JET divertor
}

\author{
Y. Corre ${ }^{1}$, P. Bunting ${ }^{2}$, J.W. Coenen ${ }^{3}$, J. Gaspar ${ }^{4}$, D. Iglesias ${ }^{2}$, G.F. Matthews ${ }^{2}$, I. Balboa ${ }^{2}$, I. \\ Coffey $^{2}$, R. Dejarnac ${ }^{5}$, M. Firdaouss ${ }^{1}$, E. Gauthier ${ }^{1}$, S.Jachmich ${ }^{3}$, K. Krieger ${ }^{6}$, R.A. Pitts ${ }^{7}$, \\ M. Rack ${ }^{3}$, S.A. Silburn ${ }^{2}$ and JET contributors ${ }^{*}$
}

EUROfusion Consortium, JET, Culham Science Centre, Abingdon, OX14 3DB, UK

1) CEA, IRFM, F-13108 Saint-Paul-Lez-Durance, France.

2) Culham Centre for Fusion Energy, Abingdon, UK

3) Forschungszentrum Jülich $\mathrm{GmbH}$, Institut für Energie und Klimaforschung Plasmaphysik, Partner of the Trilateral Euregio Cluster (TEC), 52425 Jülich, Germany

4) IUSTI UMR 7343 CNRS, Aix-Marseille University - 5 rue Enrico Fermi - 13453

Marseille - France

5) Institute of Plasma Physics CAS, Za Slovankou 3, 18200 Praha 8, Czech Republic

6) Max-Planck-Institut f. Plasmaphysik, Boltzmannstr. 2, 85748 Garching, Germany

7) ITER Organization, Route de Vinon-sur-Verdon, CS 90 046, 13067 St. Paul Lez Durance

Cedex, France

*See the author list of "Overview of the JET results in support to ITER" by X. Litaudon et al. to be published in Nuclear Fusion Special issue: overview and summary reports from the 26th Fusion Energy Conference (Kyoto, Japan, 17-22 October 2016)

\section{E-mail contact of main author: yann.corre@cea.fr}

\begin{abstract}
Tungsten (W) melting is a major concern for next step fusion devices. Two ELM induced tungsten melting experiments have been performed in JET by introducing two special target plate lamellae designed to receive excessively high ELM transient power loads. The first experiment was performed in JET in 2013 using a special lamella with a sharp leading edge gradually varying from $\mathrm{h}=0.25 \mathrm{~mm}$ to $2.5 \mathrm{~mm}$ in order to maximise the temperature rise by exposure to the full parallel heat flux. ELM-induced transient melting has been successively achieved allowing investigation of the melt motion. However, using the available IR viewing geometry from top, it was not possible to directly discriminate between the top and leading edge power loads. To improve the experimental validation of heat load and melt motion modelling codes, a new protruding $\mathrm{W}$ lamella with a $15^{\circ}$ slope facing the toroidal direction has been installed for the 2015-16 campaigns, allowing direct, spatially resolved observation of the top surface and reduced sensitivity of the analysis to the surface incidence angle of the magnetic field. This paper reports on the results of these more recent experiments, with specific focus on IR data analysis and heat flux calculations during L-mode discharges in order to investigate the behaviour of the W lamella with steady state heat load, which is a prerequisite for the more complex ELMing H-mode discharges (including both, steady and transient heat loads). It shows that, at least in L-mode, the assumption of optical heat flux projection is justified.
\end{abstract}

\section{Introduction}

Tungsten (W) melting by transient power loads, for example those delivered by edge localised modes (ELMs), is a major concern for next step fusion devices [1]. Unmitigated Type I ELMs in ITER will give rise to rapid thermal excursion that can bring the near surface temperature of the material to very high values. If the ELM power density is sufficiently high, the surface temperature can exceed the melting point for $\mathrm{W}$ and melt layer can be, partially or totally, removed [2]. At the ITER organization's request, dedicated experiments with special target 
plates have been performed in JET in order to observe the consequences of transient melting on short time scale in a real tokamak environment [3].

By virtue of its size, the amplitude of unmitigated Type I ELMs on JET can be sufficient to induce transient melting at the divertor target plates provided a deliberately misaligned element is introduced facing the full parallel plasma heat flux. A first experiment was performed in JET in 2013 using a special lamella with a sharp leading edge gradually varying from $\mathrm{h}=0.25 \mathrm{~mm}$ to $2.5 \mathrm{~mm}$ in the poloidal direction towards the high field side in order to maximise the inter-ELM temperature rise [4, 5]. Moving the outer strike point onto the sharp leading edge for about 1s enabled to reach an inter-ELM surface temperature higher than $2500^{\circ} \mathrm{C}$ allowing transient, ELM-induced melting during the subsequent duration of the pulse. ELM-induced repetitive transient melting has been successively achieved for a series of 7 very reproducible plasma discharges with plasma current of 3 MA and input power of 23 MW. However IR data analysis performed on both, reference and special lamellas, revealed significant discrepancies. The heat flux computed on the vertical side face was indeed found to be lower by a factor of 2 in H-mode and of 5 in L-mode compared to geometrical consideration. Using the available IR viewing geometry from top vertically down to the target plate surface, it was not possible to directly discriminate between the top and leading edge power loads. The method adopted and presented in [6] was to determine the parallel heat flux on the reference lamella, unperturbed by any leading edge potential effect, and use the output values on the special lamella. The heat load was derived from IR measurement on the reference lamella using the 2D (rectangular geometry), non-linear, finite difference code THEODOR [7] (step 1). The parallel plasma heat flux was derived from the geometrical flux enhancement factor and applied to the side of the special lamella (step 2) in order to simulate its thermal diffusion into the tungsten and resulting melt motion with the MEMOS code [8] (step 3). Synthetic measurements were generated to compare results from MEMOS with IR measurements on the sharp leading edge (step 4). One of the most striking results was the adhoc power reduction that was required on the leading edge to be consistent with both, spectroscopy (W vaporisation) and IR (surface temperature) experimental data. This finding stimulated the launch of an ITPA multi-device activity in support of ITER to study more carefully leading edge loading and melt dynamics [9]. As a first step, a dedicated experiment has been designed in the COMPASS tokamak to thoroughly study the power deposition on misaligned edges with submillimeter IR measurements [10]. All the power flowing into the gap is found to be deposited at the leading edge and no mitigation factor was required to explain the thermal response.

As a second step and to improve the experimental validation of heat load and melt motion modelling codes, a new protruding $\mathrm{W}$ lamella with a $15^{\circ}$ slope facing the toroidal direction has been installed in JET for the 2015-16 campaigns, allowing direct, spatially resolved observation of the top surface and reduced sensitivity of the analysis to the surface incidence angle of the magnetic field. The heat load calculation performed after the follow-up experiment follows the same stepwise procedure as depicted above (step1-4) with two main enhancements. The first one is the thermal modelling that has been further improved with forward Finite Element Method (FEM) modelling taking into account the full 3D geometry of the lamellae. The second one is the parallel heat flux density profile that has been determined along two complementary and independent axes. The first axis, presented here, is based on simulation using analytical formulation of the parallel heat flux density profile with plasma parameters (section 3). The second axis is based on direct IR inverse calculation of the parallel heat flux density profile performed with a new code ALICIA (Augmented Lagrangian Implicit Constrained Inverse Analysis). ALICA enables to get a finer mesh size at the surface 
and therefore improve heat load calculation during fast and intense events like ELMs. The final goal of the cross-modelling effort is to consolidate and reduce the boundary condition range for the simulation of the melt motion.

Reproducible L-mode discharges have been performed in both, the old and new experiments, providing, for a given parallel heat flux, IR surface temperature for three different geometries: sharp leading edge, protruding $15^{\circ}$ slope (above general tile surface) and standard shaped lamella (section 2). The forward approach includes stepwise calculations (section 3): modelling of the heat flux deposition and spatial distribution assuming particles are travelling along magnetic field in straight lines (optical projection), mapping of the magnetic field lines on the lamellas full 3D simulation of the heat diffusion in the lamellas (FEM modelling) and IR correction to simulate the spatial resolution-related effects. The amplitude of the parallel heat flux, heat flux decay length and power spreading factors are determined by iteration comparing synthetic with experimental IR data (section 4).

\section{Experimental set-up}

2.1) Geometry of the standard, former and new special lamellas

The horizontal outer target of the JET divertor is made up of 4 separate rows composed of bulk tungsten lamellas to fulfill the thermomechanical requirements during JET plasma operation. The lamellas are assembled in stacks of 24 pieces, at the top surface all of them are typically $40 \mathrm{~mm}$ high by $58 \mathrm{~mm}$ long by $5.5 \mathrm{~mm}$ wide [11]. The bulk tungsten divertor consists of four stacks, each of which is radially spaced, numbered from the inner to the outer side of the torus (A, B, C and D). In the machine, the alignment between lamellas can vary by a few tenths of millimeters due to assembly tolerances and the toroidal gap between lamellas is expected to be $1 \mathrm{~mm}$. The standard lamellas are shaped on the top surface in order to shadow their upstream edges from the parallel heat flux. The shaping automatically leads to a reduction of the wetted top surface area, due to magnetic shadowing [12], combined with an increase of the deposited heat flux. The shaping is an important feature which makes the IR data analysis sensitive to both the relative alignment between lamellas and to the inclination of the magnetic field lines at the shaped surface. The heat flux deposited on the shaped lamella is, therefore, non-uniform in both, toroidal (due to the variation of the inclination of the magnetic field line and magnetic shadowing) and poloidal (due to power decay length in the SOL and power spreading factor in the private flux region) directions respectively. The 2D simplification proposed in [6] for the shaped lamella has been further enhanced to full 3D heat load and heat diffusion modelling in the present analysis.

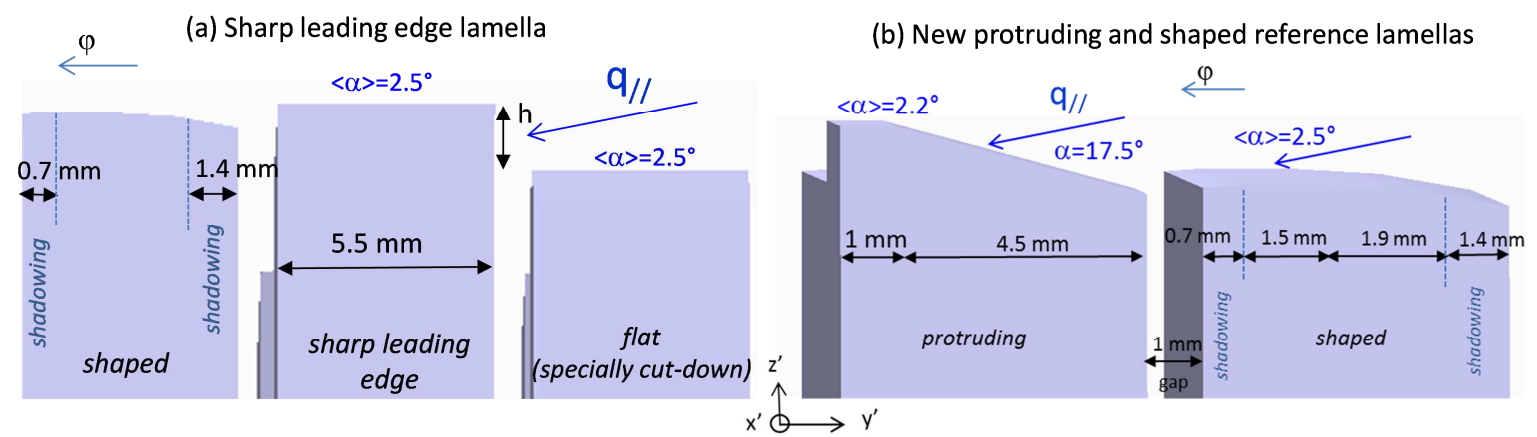

Figure 1: Toroidal cross section of (a) former lamella with sharp leading edge and (b) new lamella with $15^{\circ}$ slope protruding part and standard lamella including wetted and shadowing areas. 
For the purpose of the first tungsten melt experiment, one of the inboard stacks (labelled stack A) has been modified to expose the side of one special lamella. Eight lamellas in front of the special lamella have been specially cut down in order to create the exposed leading edge by the preceding recessed surface. The first special lamella has no shaping, the top surface is therefore completely flat and the exposed side face is sharp with a height varying from $0.25 \mathrm{~mm}$ at the low field side (LFS) up to $2.5 \mathrm{~mm}$ on the high field side (HFS). In the follow-up tungsten melt experiment, the new sloped special lamella is made up of a protruding $15^{\circ}$ inclined surface, $4.5 \mathrm{~mm}$ large, with a $1 \mathrm{~mm}$ flat top in order to avoid sharp corner. To prevent unintended melting in other experiments, the protruding part is restricted to the last $20 \mathrm{~mm}$ on the inner side of the lamella (stack A). The rest of the lamella, on the outer side, is shaped identically to the standard ones. The protruding and shaped parts of the lamella are separated by a keyhole of $0.5 \mathrm{~mm}$ width and $10 \mathrm{~mm}$ depth. The keyhole offers a clear advantage for the heat transfer modelling through the lamella, since it behaves like a thermal barrier which separates the protruding from the standard parts of the lamella. Figure 1 shows the toroidal cross-sections of the former (a) and new special lamellas (b) with the parallel heat flux along the magnetic field. The geometry is defined in the cylindrical machine coordinates: $\{\mathrm{R}, \varphi, \mathrm{z}\}$ where $\mathrm{R}$ is the radial direction, $\varphi$ the toroidal direction and $\mathrm{z}$ the vertical direction. The local frame of the lamella is also used $\left\{x^{\prime}, y^{\prime}, z^{\prime}\right\}$, where $x^{\prime}$ and $y^{\prime}$ correspond to the poloidal and toroidal directions as shown in figure 1.

\section{2) ELM-induced repetitive transient melting experiment}

In order to approach transient ELM-induced melting cautiously, the two experiments, based on former and new special lamellas, have been conducted in three steps. The first step aimed to characterize the thermal behavior of the special lamellas. To that end, L-mode discharges with the outer strike point moving from (unmodified) stack B to stack A (including the special lamella) have been run with different strike line positions $\left(\mathrm{x}_{0}\right)$ and excursion times on stack $\mathrm{A}$ in order to control the power and energy deposited on the special lamella and hence its temperature (so to avoid unintended and steady melting). During the former tungsten melt experiment, the typical strike-line location was chosen close to the center of the lamella leading to an exposure height lying between $\Delta \mathrm{h} \sim 1.3 \mathrm{~mm}$ (\#84513) and $1.5 \mathrm{~mm}$ (\#84514). In the second step the ELM energy was incrementally adjusted in order to reach a transient temperature rise of $1000^{\circ} \mathrm{C}$ for each single ELM (requiring ELMs in the $\mathrm{GW} / \mathrm{m}^{2}$ parallel heat flux density range). The strike point was positioned on stack B and moved toward stack A for a limited amount of time in order to get the temperature rise for a couple of ELMs. The fueling rate was successively decreased in order to achieve a regular train of low frequency type I ELMs. Regular type I ELMs with about $300 \mathrm{~kJ}$ energy release and $\mathrm{f}_{\mathrm{ELM}} \sim 30 \mathrm{~Hz}$ have been achieved in both, the former and new tungsten melt experiments, with plasma current of $\mathrm{Ip}=3 \mathrm{MA}$, toroidal magnetic field $\mathrm{B}_{\mathrm{t}}=2.86 \mathrm{~T}$ and heating power $\mathrm{P}^{\mathrm{NBI}}>20 \mathrm{MW}$ [4]. The third and final step aimed at inducing transient tungsten melting by extending the excursion time on the special lamella. 7 subsequent discharges including about 150 transient melt events and 2 discharges including about few tenths of transient melt events have been achieved in the former and new melting experiment respectively.

\section{3) IR thermography system}

The infrared (IR) thermography system which monitors the surface temperature around the special lamellas consists of a $100 \mathrm{~mm}$ objective lens and IR camera installed in the upper port of the machine (KL9A). This provides a vertical view down to the horizontal divertor plate surfaces with a pixel size of $\sim 1.7 \mathrm{~mm}$ (projection of the pixel into the object plane). The IR camera is a FLIR SC7500 filtered to observe the middle IR wavelength range $(3.1-4.7 \mu \mathrm{m})$ with an array of $240 \times 320$ pixels and $30 \mu \mathrm{m}$ pixel pitch. The transfer function 
of the IR instrument has been modelled by a Gaussian distribution function. The standard deviation $(\sigma)$ of the JET instrument is deduced from the laboratory measurements [13] using the magnification of the system, we find $\sigma=1 \mathrm{~mm}$ for a pixel size of $1.7 \mathrm{~mm}$.

\section{4) Reproducible L-mode discharges}

The most critical aspect of the analysis of the $\mathrm{W}$ melting experiment is the calculation of the heat loads from the lamella temperatures provided by the IR thermography vertical viewing system. Without the correct input heat loads, simulations of the transient melt motion, the key result being sought, cannot be reliably benchmarked. To this end, reproducible L-mode discharges with $\mathrm{I}_{\mathrm{p}}=2.45 \mathrm{MA}, \mathrm{B}_{\mathrm{T}}=2.6 \mathrm{~T}, \mathrm{P}_{\mathrm{NBI}}=2 \mathrm{MW}$ and $2 \mathrm{~s}$ lamella exposure have been performed in both the old and new experiments, providing, for a given parallel heat flux, the IR surface temperature for three different geometries: (a) sharp leading edge (\#84514), (b) protruding $15^{\circ}$ slope (\#89612) and (c) standard shaped lamella (\#89612). The outer strike point is positioned on stack $\mathrm{B}\left(\mathrm{R}_{\mathrm{OSP}} \sim 2.66 \mathrm{~m}\right)$ at $\mathrm{t}=50 \mathrm{~s}$ when additional power is switched on, and start to move toward stack $A\left(R_{O S P} \sim 2.6 \mathrm{~m}\right)$ at $t=55.5 \mathrm{~s}$. The plasma sweeping duration is about $1 \mathrm{~s}$, thus the strike point is steadily positioned on stack A from $\mathrm{t}=56.5 \mathrm{~s}$ until $58.5 \mathrm{~s}$ (see Figure 2).

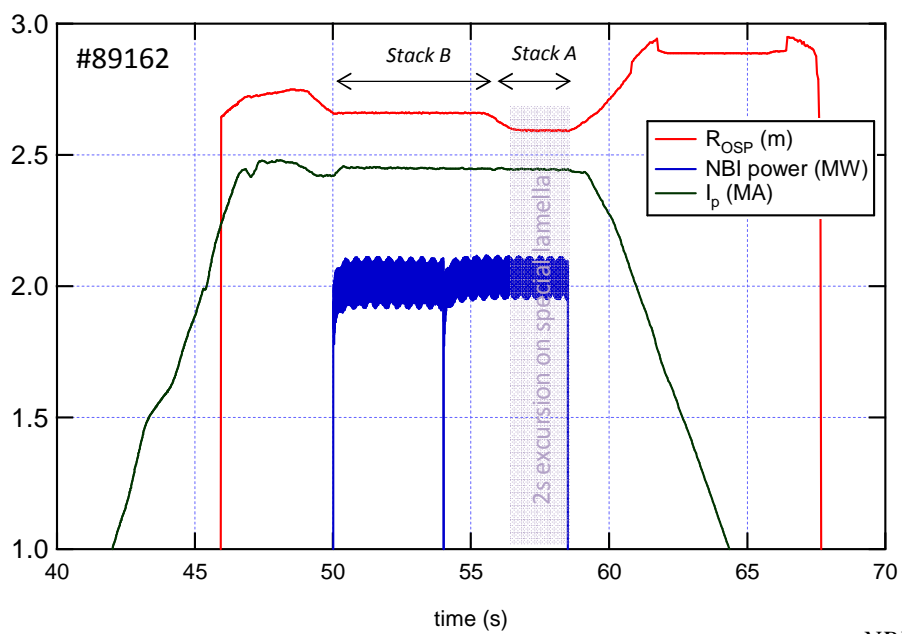

Figure 2 : Outer Strike Point radial location $\left(R_{O S P}\right), N B I$ power $\left(P^{N B I}\right)$ and plasma current $\left(I_{p}\right)$ as function of time for \#89162 (L-mode experiment)

Figure 3 displays the strike point location, visualized with the software PFCFlux [14] (Plasma Facing Components flux), on both, reference and protruding special lamellas. The main PFCFlux inputs are the 3D outlines of the horizontal outer divertor plate module including the lamellas (CAD drawing) and 3D magnetic field given by the magnetic equilibrium. This shows that, due to geometrical consideration, the strike point is shifted by about $1.2 \mathrm{~mm}$ on the inner side on the flat top part of the protruding lamella compared to the reference lamellas. This shift has been introduced in the following thermal modelling. 


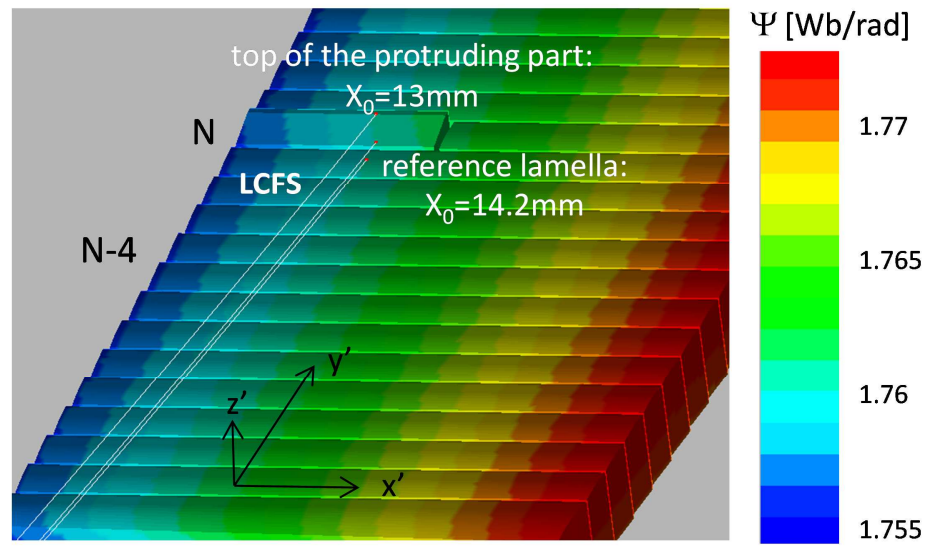

Figure 3: Field line tracing performed with PFCFlux software (EFIT magnetic equilibrium, \#89162@ $t=58$ s). Field lines are chosen on the last closed magnetic flux surface $(\Psi=1.759 \mathrm{~Wb} / \mathrm{rad})$.

\section{Heat load calculation and thermal behavior of the lamellas}

3.1) Modelling of the heat load

Assuming that particles are travelling along the magnetic field in straight lines (ballistic guiding-center approach), the heat load distribution can be computed with the projection of the parallel $\left(\mathrm{q}_{/ /}\right)$heat flux for each of the three geometries:

$$
q_{n}=q_{/ /} \cdot \sin \alpha+q_{B G}
$$

where $\alpha$ is the angle between the magnetic field line with the surface and $q_{B G}$ represents the background heat flux (including cross-field heat flux, plasma radiation and neutral particles coming from charge exchange). The background heat flux is supposed to be homogeneous in the radial direction. In this following analysis, the background heat flux to the top surface of the lamella has been set to $q_{B G}=0.3 \mathrm{MW} \cdot \mathrm{m}^{-2}$, which represents few percent of the peak heat load on the protruding and less than $10 \%$ on the reference lamellas, respectively. The incident angle and magnetic shadowing pattern are computed with PFCFlux. The simulations have been performed based on two reproducible L-mode discharges, \#84514 (sharp leading edge) and \#89612 (protruding $15^{\circ}$ slope), during the excursion on stack A (@ $\mathrm{t}$ $=58 \mathrm{~s})$.
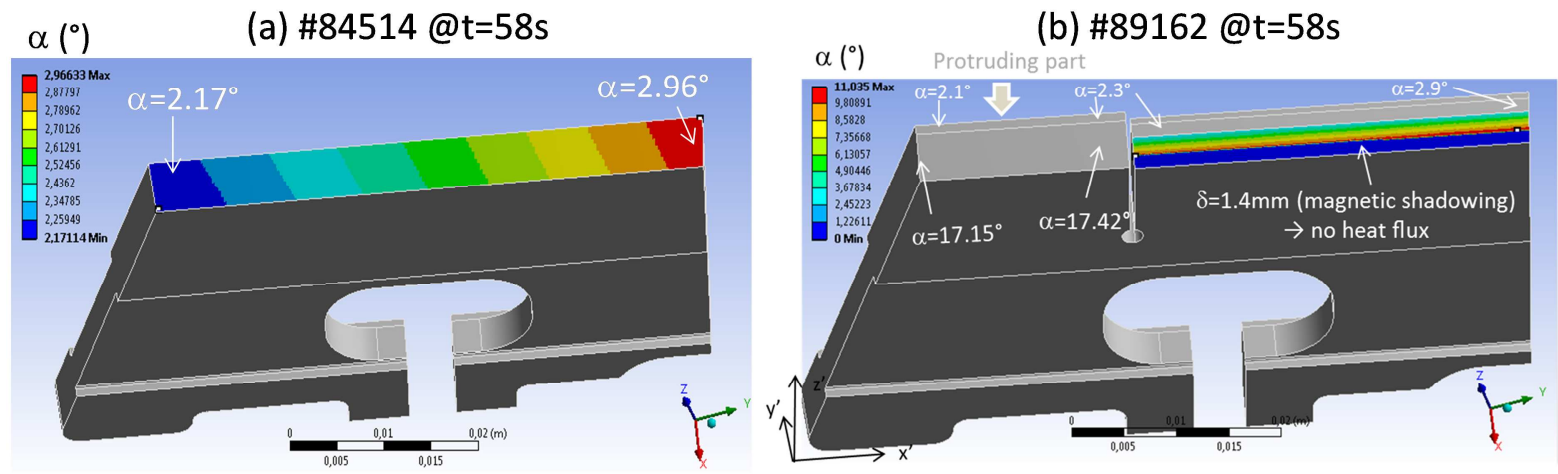

Figure 4: Angle between magnetic field lines with top surfaces computed with the PFCflux software (\#89162@ $t=58 \mathrm{~s}$ ), on protruding (top and $15^{\circ}$ slope surfaces) and shaped parts respectively. The magnetic shadowing from the previous lamella is found to be about $\delta=1.4 \mathrm{~mm}$. 
Figure 4 shows the spatial distribution of incident angle and magnetic shadowing on the former (a) and new special (b) lamellas. On the flat top of the sharp leading edge lamella, the incident angle varies between $2.17^{\circ}$ on the inner side and $2.96^{\circ}$ on the outer side. On the flat top of the protruding lamella, the incident angle varies between $2.1^{\circ}$ on the inner side and $2.3^{\circ}$ near the keyhole, while it varies between $17.15^{\circ}$ on the inner and $17.42^{\circ}$ near the keyhole on the $15^{\circ}$ slope part. On the flat top part of the standard geometry, $\alpha$ varies between $2.3^{\circ}$ near the keyhole and $2.9^{\circ}$ on the outer side, while $\alpha$ varies between $2.8^{\circ}$ and $10^{\circ}$ on the shaped part. The magnetic shadowing is found to be $\delta=1.4 \mathrm{~mm}$ on the shaped part of the lamella (assuming that the previous lamella is perfectly aligned with the special lamella). The parallel heat flux density radial distribution is expressed with the heuristic formulation proposed in [15]:

$$
q_{/ /}(x)=A \exp \left[\left(\frac{s}{2 \lambda_{q}}\right)^{2}-\frac{x-x_{0}}{\lambda_{q} f_{x}}\right] \operatorname{erfc}\left(\frac{s}{2 \lambda_{q}}-\frac{x-x_{0}}{S f_{x}}\right)
$$

where $\mathrm{x}$ is the target coordinate, $\mathrm{x}_{0}$ is the strike point location, $\lambda_{\mathrm{q}}$ is the power decay length inside the scrape off layer, $S$ is the power spreading factor in the private flux region and $f_{x}$ is the magnetic flux expansion from the outboard mid-plane to the component. A is the experimental heat flux factor computed iteratively in order to match the IR data. The peak heat load and power decay length are determined by iteration comparing synthetic with experimental IR data on the three different geometries. Figure 5 shows the parallel heat flux

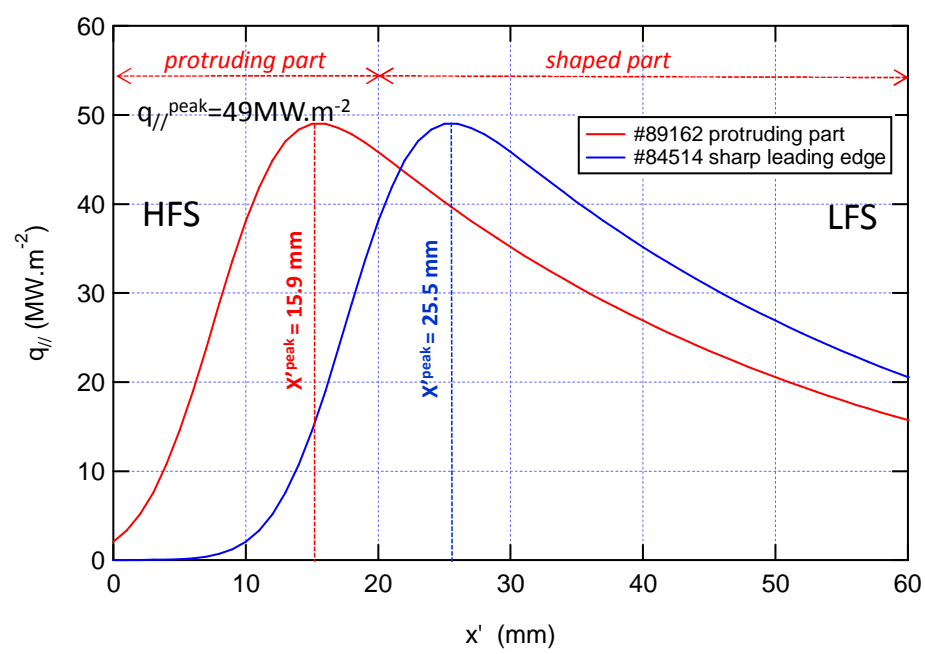

Figure 5: Parallel heat flux density radial distribution used for \#84514 and \#89162 during the excursion on stack $A$, assuming $f_{x}=6.2, S=1 \mathrm{~mm}, \lambda_{q}=6 \mathrm{~mm}$ and $q_{/ /}^{\text {peak }}=49 \mathrm{MW} \cdot \mathrm{m}^{-2}$.

density radial distribution, for the two strike point locations on stack A ( $x^{\prime \text { peak }}=15.9 \mathrm{~mm}$ for $\# 89162$ and $x^{\prime \text { peak }}=25.5 \mathrm{~mm}$ for \#84514), obtained with $\mathrm{f}_{\mathrm{x}}=6.2$ derived from the magnetic equilibrium, $\lambda_{\mathrm{q}}=6 \mathrm{~mm}$ and $\mathrm{S}=1 \mathrm{~mm}$ which are determined after the iteration process with the IR data.

3.2) 3D thermal modelling and synthetic IR data

Thermal modelling has been performed using Finite Element Method (FEM) and 3D geometry of the lamellas (ANSYS V16). The heat load is computed with the surface projection of the parallel heat flux density as depicted in the previous section. The heat load temporal variation is approximated in 7 discrete time slices during the sweeping of the strike point from stack B to A (with $6.5 \mathrm{~mm}$ shift every $100 \mathrm{~ms}$ ) and set stationary during the $2 \mathrm{~s}$ excursion on stack A (figure 6). 


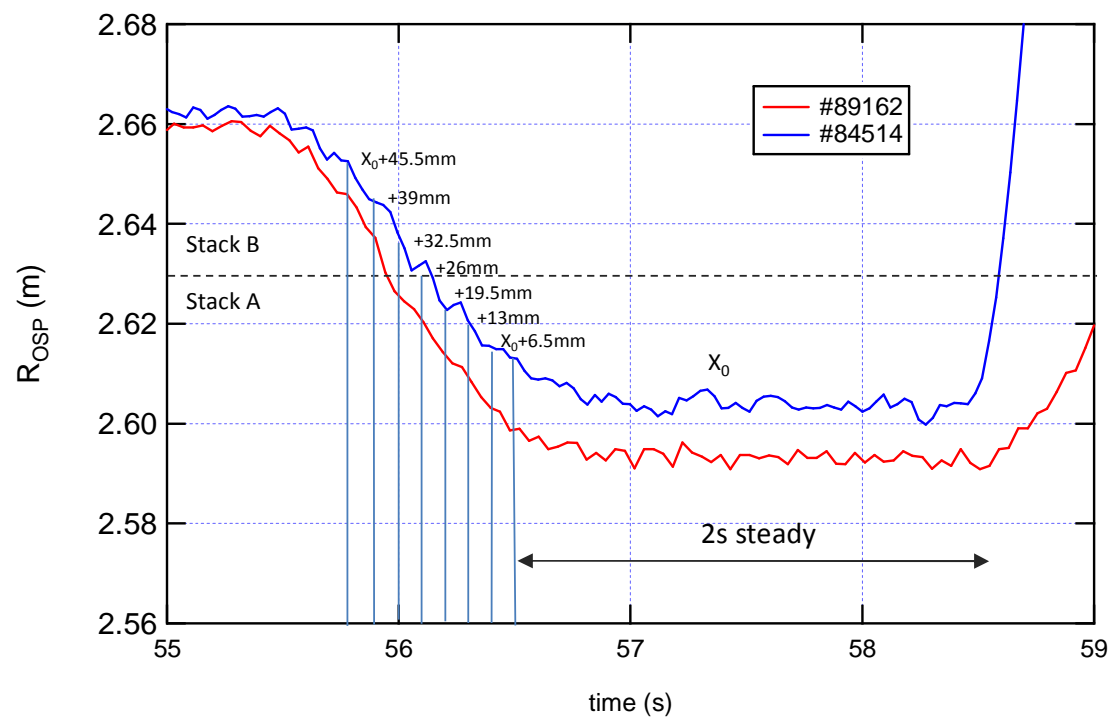

Figure 6: Outer Strike Point radial location $\left(R_{O S P}\right)$ as function of time for the former (blue) and new experiments (red). Discretization of the heat load temporal variation during sweeping of the strike point.

Temperature distributions are convoluted with the Modulated Transfer Function (MTF) of the instrument to simulate the spatial resolution related effects. The total MTF can be modelled by a Gaussian function in frequency space with $\sigma$ being the standard deviation. The standard deviation of the IR camera has been determined by a laboratory blackbody-slit experiment as described in [13]. In the JET configuration (KL9A camera), the standard deviation is extrapolated from the blackbody-slit experiment, we found $\sigma=1 \mathrm{~mm}$, which is equivalent to $\sim 60 \%$ of the pixel size in the object plane.

\section{4) Experimental results}

4.1) Results on the new protruding and shaped lamellas (follow-up experiment)

On the protruding part, the peak parallel heat flux is found to be $q_{/ /}^{\text {peak }}=49 \mathrm{MW} \cdot \mathrm{m}^{-2}$ with $\pm 5 \%$ relative error, which is attributed mainly to the uncertainty about the SP position (of the order of the IR pixel size, $\pm 1.7 \mathrm{~mm}$ ). Assuming $q_{B G}=0.3 \mathrm{MW} \cdot \mathrm{m}^{-2}$ and according to equation (1), this corresponds to a peak heat load $q_{n}^{\text {peak }} \sim 14.8 \mathrm{MW} \cdot \mathrm{m}^{-2}$ on the $15^{\circ}$ slope and $q_{n}^{\text {peak }} \sim 2.1-2.8 \mathrm{MW} \cdot \mathrm{m}^{-2}$ on the flat top part of the reference lamella inboard/outboard sides respectively (with again $\pm 5 \%$ relative error due to SP position). To get an overall agreement on both, protruding and shaped parts of the lamella, we iterate on the heat flux decay length and power spreading factor values. The experimental surface temperature poloidal distribution is properly reproduced with $\lambda_{\mathrm{q}}=6 \mathrm{~mm}$ and $\mathrm{S}=1 \mathrm{~mm}$. However, the amplitude of the simulated temperatures obtained on the shaped part, assuming the theoretical magnetic shadowing of $\delta=1.4 \mathrm{~mm}$, are about $15 \%$ lower than experimental data. This is attributed to the magnetic shadowing which is very sensitive to vertical misalignment between two adjacent lamellas (see table 1). The magnetic shadowing has been recomputed on the new special lamella $(\mathrm{N})$ assuming $0.1 \mathrm{~mm}$ downward misalignment compared to the previous lamella $(\mathrm{N}-1)$, we found $\delta=0.9 \mathrm{~mm}$. A new run has therefore been performed with reduced magnetic shadowing area $(\delta=1.4 \rightarrow 0.9 \mathrm{~mm})$. The discrepancy between simulation and experimental data on the shaped part then decreases from $15 \%$ (assuming $\delta=1.4 \mathrm{~mm}$ ) down to few $\%$ (assuming $\delta=0.9 \mathrm{~mm}$ ). 


\begin{tabular}{|c|c|c|c|c|c|c|}
\hline & $\mathrm{N}$ (special) & $\mathrm{N}-1$ & $\mathrm{~N}-2$ & $\mathrm{~N}-3$ & $\mathrm{~N}-4$ & $\mathrm{~N}-5$ \\
\hline$T^{\max }$ & $514^{\circ}$ & $480^{\circ}$ & $490^{\circ}$ & $470^{\circ}$ & $509^{\circ}$ & $485^{\circ}$ \\
\hline$T^{\max }-<T^{\max }>$ & $23^{\circ}$ & $-11^{\circ}$ & $-1^{\circ}$ & $-21^{\circ}$ & $18^{\circ}$ & $-6^{\circ}$ \\
\hline$T^{\max }-<T^{\max }>$ & $+8.8 \%$ & $-4.2 \%$ & $-0.4 \%$ & $-8 \%$ & $+7 \%$ & $-2.3 \%$ \\
\hline$\Delta T$ & & & & & & \\
\hline
\end{tabular}

Table 1: Temperature scattering measured on 6 successive lamellas when heating is switched off (@ $t=58.5 \mathrm{~s})$. The ratio between the temperature scatterings with the averaged heating is attributed to the misalignment between lamellas.

Figure 7 shows the simulated and experimental IR data (\#89162) in the poloidal (a) and toroidal (b) directions when the NBI heating is switched off (@ $\mathrm{t}=58.5 \mathrm{~s}$ ). The two simulated temperature profiles are in good agreement with IR data profiles, on both, protruding and shaped parts (assuming $\delta=0.9 \mathrm{~mm}$ ). In the poloidal direction ${ }^{1}$, the spatial resolution related effects are significant inside an area limited by \pm 2 IR pixels around the keyhole, around the gap between the two stacks and negligible elsewhere. In the toroidal direction, the spatial resolution related effect is small (about 3\% of the global heating) in the center of the lamella and significantly higher on the sides (near the gaps). In the center of the protruding lamella $\left(y^{\prime}=2.75 \mathrm{~mm}\right)$, the FEM modelling gives $\mathrm{T}^{\max }($ true $)=1180^{\circ} \mathrm{C}$ while simulated and experimental IR data are found to be $\mathrm{T}^{\max }$ (synthetic) $=1150^{\circ} \mathrm{C}$ and $\mathrm{T}^{\max }(\mathrm{IR})=1135^{\circ} \mathrm{C}$ respectively.
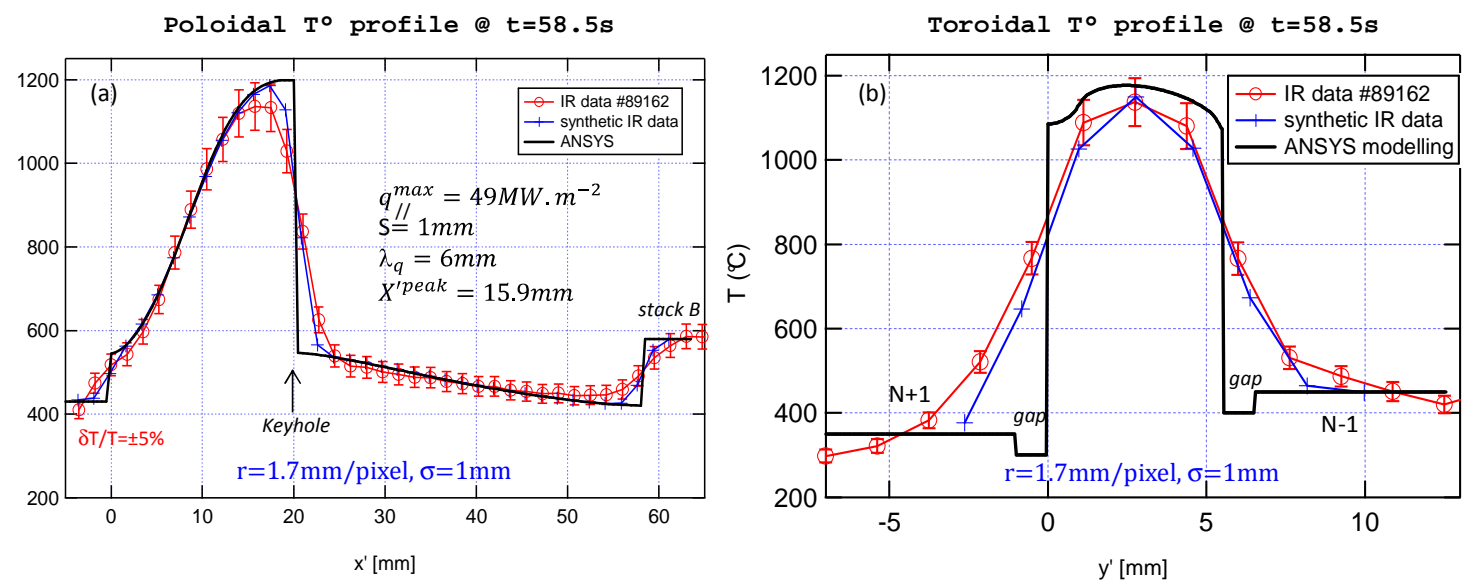

Figure 7: Poloidal (a) and toroidal (b) temperature profiles on the new protruding lamella. IR data assuming $\pm 5 \%$ error bars (red circles), ANSYS modelling (black line) and synthetic IR data (blue crosses).

Figure 8 shows the time evolution of the surface temperature (without convolution with the MTF) at the peak heat load location $\left(x^{\prime \text { peak }}=15.9 \mathrm{~mm}\right)$. Simulated and experimental surface temperatures are in good agreement especially during the heating phase (the simulation is slightly higher than experimental data due to the expected $2 \mathrm{D}$ spatial resolution effects).

\footnotetext{
${ }^{1}$ In this paper, the convolution with the MTF is always 1D, in the poloidal or toroidal directions, but not both in the same time.
} 


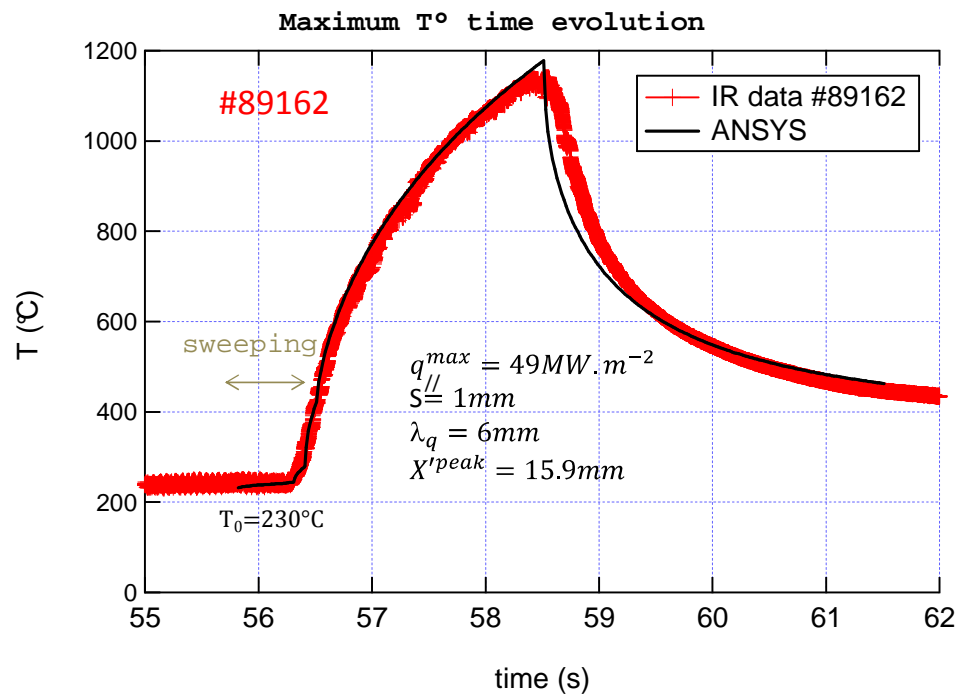

Figure 8: Maximum of the surface temperature as function of time: ANSYS modelling (black line) and IR data (red crosses).

The same plasma parameters $\left(q_{/ /}^{\text {peak }}=49 M W \cdot \mathrm{m}^{-2}, \lambda_{q}=6 \mathrm{~mm}, S=1 \mathrm{~mm}\right.$ and $q_{B G}=0.3 \mathrm{MW} \cdot \mathrm{m}^{-2}$ ) have been applied to the shaped reference geometry with the strike point shifted by $\delta x^{\prime}=0.6 \mathrm{~mm}$ (half of the value computed with the PFCFlux code, see figure 3 ) on the outer side, thus for reference shaped lamella we have used $x^{\prime \text { peak }}=16.5 \mathrm{~mm}$. Simulated (with magnetic shadowing $\delta=0.9 \mathrm{~mm}$ ) and experimental IR data (\#89162) have been compared on the reference shaped lamella $(\mathrm{N}-4)$ in the poloidal directions when the NBI heating is switched off (@ $@=58.5 \mathrm{~s}$ ). We obtain a good agreement on the full lamella length (with a discrepancy of less than $5 \%$ when $x^{\prime}$ lies between 0 and $50 \mathrm{~mm}$ and less than $10 \%$ on the low field side of the lamella that is more sensitive to the strike point movement, when $x^{\prime}$ lies between 50 and $59 \mathrm{~mm}$ ), which means that the set of plasma parameters we use is consistent with both, protruding and shaped geometries.

4.2) Results on the sharp leading edge special lamella (former experiment)

The same plasma parameters $\left(q_{/ /}^{\text {peak }}=49 M W \cdot \mathrm{m}^{-2}, \lambda_{q}=6 \mathrm{~mm}, S=1 \mathrm{~mm}\right.$, and $q_{B G}=0.3 \mathrm{MW} \cdot \mathrm{m}^{-2}$ ) have finally been applied, using direct modelling without any iteration, to the sharp leading edge geometry with the peak heat flux shifted by $\sim 1 \mathrm{~cm}$ outboard as computed with EFIT magnetic equilibrium, $x^{\prime \text { peak }}=25.5 \mathrm{~mm}$. Figure 9 (left) shows the 3D temperature map simulated with ANSYS when the NBI heating is switched off (@ $\mathrm{t}=58.5 \mathrm{~s}$ ). At the peak heat flux location, the misalignment is $\mathrm{h}=1.5 \mathrm{~mm}$ and the peak temperature on the corner is $1880^{\circ} \mathrm{C}$ (ANSYS modelling). With such a misalignment, the toroidal temperature gradient is very high $\left(\sim 150^{\circ} \mathrm{C} / \mathrm{mm}\right.$ on the flat top surface and even higher in the gap region) and the spatial resolution effect is maximum. Figure 9 (right) shows the simulated and experimental IR data (\#84514) in the toroidal directions, near the peak heat flux, when the NBI heating is switched off ( @ t=58.5s). The maximum of the synthetic IR data is found to be $1285^{\circ} \mathrm{C}$, which is slightly below the experimental IR data $\left(1365^{\circ} \mathrm{C}\right)$. The resulting $80^{\circ} \mathrm{C}$ temperature discrepancy between synthetic and IR data can be explained with either a small shift of the SP in the inboard side or a slightly higher parallel heat flux density (supported by the NBI heating power which is slightly higher during shot \#84514 than \#89612). 

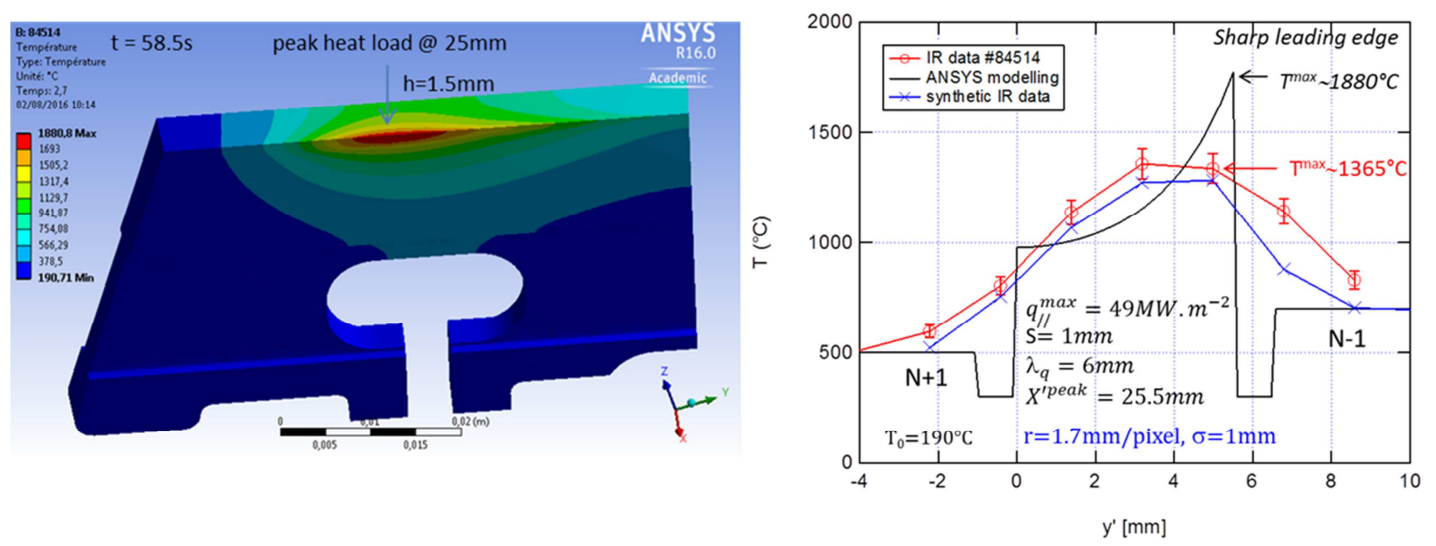

Figure 9: ANSYS simulation with the sharp leading edge geometry when power is switched off @ $\mathrm{t}=58.5 \mathrm{~s}$. Toroidal temperature profile on the sharp leading edge lamella at $x^{\prime \text { peak }}=25.5 \mathrm{~mm}$ : IR data assuming $\pm 5 \%$ error bars (red circles), ANSYS modelling (black line) and synthetic IR data (blue crosses).

In summary, no mitigation factor is required to match the IR data during L-mode operation. Using the same set of plasma parameters for the three different geometries, all simulations are in good agreement with the IR data provided in two reproducible but separate plasma experiments. The remaining differences are lower than the global uncertainties (strike point location at \pm 1 IR pixel, assuming IR data at $\pm 5 \%^{2}$ ). The total power loads computed for each of the three geometries are given in table 2. Protruding and leading edge special lamellas receive, accordingly to the optical projection, two and three times higher heat load than shaped lamellas during L-mode plasma discharge, respectively.

\begin{tabular}{|c|c|c|c|}
\hline & $\begin{array}{c}\text { Shaped reference N-4 } \\
(\# 89162)\end{array}$ & $\begin{array}{c}\text { Special protruding } \\
(\# 89162)\end{array}$ & $\begin{array}{c}\text { Special leading edge } \\
\text { (\#84514) }\end{array}$ \\
\hline Total power [W] & 756 & 1373 & 2345 \\
\hline
\end{tabular}

Table 2: Total power received by the lamellas during the 2 s excursion on stack $A$.

\section{Conclusion}

Reproducible L-mode discharges have been performed in the first and second JET transient tungsten melt experiments, providing, for a given parallel heat flux, IR surface temperatures for three different geometries: sharp leading edge, protruding $15^{\circ}$ slope and standard shaped lamella. Forward approach based on full 3D heat load and thermal modelling of the lamellas has been performed assuming an optical projection of the parallel heat flux to the lamella surface, together with a specific IR correction to simulate spatial resolution related effects. Using the same model and same plasma parameters, good agreement is obtained for all three geometries, validating the assumption of optical heat load projection and providing a solid basis for the more complex ELMing H-mode conditions. The follow-up experiment and associated analysis provide a consistent description of the heat load received by the JET standard and special lamellas with no power mitigation factor on sharp leading edge. The new insight relies mainly on two key points: On the one hand the new geometry based on a protruding $15^{\circ}$ slope special lamella is less sensitive to magnetic shadowing and to the inclination of the magnetic field lines with the surface. Moreover the IR camera views directly the $15^{\circ}$ slope exposed to the increased heat flux. On the other hand, the forward modelling combined with the full 3D description of the plasma heat load and heat diffusion in the

\footnotetext{
${ }^{2}$ This assumption does not take into account the uncertainty related to the emissivity of the bulk tungsten which is unknown in JET.
} 
lamella results in a significantly improved simulation accuracy. Further studies are required to investigate the heat load during ELMs and $\mathrm{W}$ melt dynamic in the JET transient melt experiment.

\section{Acknowledgments:}

This work has been carried out within the framework of the EUROfusion Consortium and has received funding from the Euratom research and training programme 2014-2018 under grant agreement No 633053. The views and opinions expressed herein do not necessarily reflect those of the European Commission and ITER Organization.

\section{References:}

[1] A. Loarte, et al., Nucl. Fusion 47, S203 (2007).

[2] G. Federici et al. Plasma Phys. Control. Fusion 45 (2003) 1523-1547

[3] R. Pitts et al. Journal of Nuclear Materials 438 (2013) S48-S56

[4] J.W. Coenen et al. Journal of Nuclear Materials 463 (2015) 78-84

[5] J.W. Coenen et al. Nucl. Fusion 55 (2015), 023010

[6] G. Arnoux et al. J. Nucl. Mater. 463 (2015), 415

[7] A. Herrmann et al. Plasma Phys. Control. Fusion. 37 (1995) 17-29

[8] B. Bazylev et al. J. Nucl. Mater. 390-391 (2009) 810-813

[9] R. Pitts et al. J. Nucl. Mater, in press. (2017)

[10] R. Dejarnac et al. Nuclear Materials and Energy 000 (2016) 1-5

[11] Ph. Mertens et al. Fusion Engineering and Design 84 (2009), 1289-1293

[12] S. Grigoriev et al. Fusion Engineering and Design 84 (2009) 853-858

[13] Y. Corre et al. Nucl. Fusion 57 (2017) 016009 (9pp)

[14] M. Firdaouss et al. J. Nucl. Mater. 438 (2013) S536-S539

[15] T. Eich et al. 2013, J. Nucl. Mater. 438 S72-S77 